\title{
Role of Polycarboxylate-ether superplasticizers on cement hydration kinetics and microstructural development
}

\author{
L. Valentini, M. C.Dalconi and G.Artioli \\ University of Padua, Department of Geosciences, Padua, Italy
}

\begin{abstract}
Polycarboxylate-ether (PCE) superplasticizers are a fundamental constituent of modern cementbased materials due to their impact on the rheology of the fresh mix and mechanical performance of the hardened material. The effect of PCEs on cement hydration kinetics has been known since their introduction in the early 1980s. However, detailed knowledge of the role played by PCE macromolecules on the basic mechanisms of cement hydration (dissolution, diffusion, precipitation) is still lacking. A better understanding of how such mechanisms are influenced by the addition of PCE is no doubt beneficial to the design of novel superplasticizing admixtures. Here, I report on some recent findings about the role of PCE superplasticizers on cement hydration kinetics and microstructural development. The interaction between PCE and $\mathrm{C}_{3} \mathrm{~S}$ pastes was investigated by an ad-hoc kinetic model based on a combination of generalized forms of the Avrami and BNG (Boundary Nucleation and Growth) models. The model is used to fit the rate of C-S-H precipitation measured by in-situ X-ray powder diffraction combined with mass balance calculations. The results show that a switch from heterogeneous to homogeneous C-S-H nucleation occurs in the presence of PCEs and that the C-S-H growth rate decreases proportionally to the amount of PCE used. The predicted switch to homogeneous nucleation is in agreement with experimental results obtained by XRD-enhanced micro-tomography imaging, showing that, in the presence of PCE, C-S-H preferentially forms in the pore space rather than at the surface of clinker particles.
\end{abstract}

\section{Introduction}

The introduction of admixtures for the control of concrete rheology has represented a significant technological advance. Starting from the first generation of superplasticizers, up to the modern polycarboxylateether (PCE) molecules, which currently dominate the market, the capability of such admixtures to allow water reduction and better mechanical performance has progressively increased. By providing a detailed description of the mechanisms by which PCE admixtures exert their dispersing action, it has been possible to finetune their structure with the aim of providing the desired behaviour as a function of the specific application. For example, the synthesis of PCE macromolecules having different anionic functions can lead to improved affinity for clinker surfaces [1]. However, there are specific situations in which the action of PCE admixtures is hampered. These include the use of PCE with concrete made with cements having large fractions of orthorhombic $\mathrm{C}_{3} \mathrm{~A}$ [2] and with aggregates having a significant content of clay minerals [3].

From the point of view of hydration kinetics, although the delaying effect of PCE molecules has been well documented, it is still not clear what are the basic mechanisms that control such a delay. Therefore, a detailed investigation of such mechanisms is necessary to achieve a better control of the hydration kinetics in the presence of PCE.

To date, the main invoked mechanisms for the delay of the hydration kinetics are the hindering of dissolution of the clinker phases or nucleation and growth of the hydration products.

Here, I present an investigation of the hydration kinetics of a model $\mathrm{C}_{3} \mathrm{~S}$ cement, in the presence of different PCE molecules. In-situ XRD data are combined with mass balance calculations and kinetic modelling, with the aim of providing a quantitative description of the modes and rates of C-S-H nucleation and growth.

\section{Materials and methods}

Cement pastes were prepared by mixing $\mathrm{C}_{3} \mathrm{~S}$ with deionized water at $\mathrm{w} / \mathrm{c} 0.5$, for 1 minute, using a vertical stirrer. PCE admixtures (having 17 repeat units in the side-chains and 3 to 4 repeat units in the backbone for each segment) were added directly to the mix water, at dosages of $0.05 \%$ and $0.10 \%$ by mass of $\mathrm{C}_{3} \mathrm{~S}$. 


\subsection{XRD measurements}

$\mathrm{C}_{3} \mathrm{~S}$ reaction kinetics, for the blank paste and in the presence of PCE, were monitored by means of in-situ Xray powder diffraction, using $0.5 \mathrm{~mm}$ borosilicate glass capillaries filled with the sample. The measurements were performed in time-resolved mode for 20 hours, with a time resolution of 20 minutes and with a constant temperature of $23{ }^{\circ} \mathrm{C}$. Quantitative phase analyses of the diffraction patterns were performed using the software Topas 4.1 (Bruker). The time-dependent volume of C-S-H was calculated using a mass balance algorithm [4], as implemented in the software RieCalc 3.2 [5].

\subsection{Kinetic Model}

The kinetics of C-S-H precipitation have been modelled by combining generalized forms of the Johnson-MehlAvrami-Kolmogorov (JMAK) and Boundary Nucleation and Growth (BNG) models [6,7]. The following kinetic equation was used to fit the experimental C-S-H rate of precipitation obtained by XRD:

$\left.X(t)=A\left\{1-\exp [-X(t))_{e}^{h o m}-X(t)_{e}^{h e t}\right]\right\}$

Where $X$ is the volume fraction of C-S-H precipitated at time $t, \mathrm{~A}$ is a scaling constant and the two terms inside the exponential are the extended volume fractions (representing the theoretical volume that would form ifthere was no overlap among particles) formed by homogeneous and heterogeneous nucleation respectively, given by the two following equations:

$$
\begin{aligned}
& X(t)_{e}^{h o m}=\frac{4}{3} \pi N_{v} \Gamma^{3}(t) \\
& X(t)_{e}^{h e t} \\
& =O_{B} \int_{0}^{1} \Gamma(t)\left\{1-\exp \left[-\pi N_{S} \Gamma^{2}(t)\left(1-u^{2}\right)\right]\right\} d u
\end{aligned}
$$

Where $\mathrm{N}_{\mathrm{v}}$ and $\mathrm{N}_{\mathrm{s}}$ are the number of nuclei formed by homogeneous and heterogeneous nucleation respectively, $\Gamma$ is the time-dependent growth rate $\mathrm{O}_{\mathrm{B}}$ is the amount of cement surface per unit volume, $u$ is an auxiliary variable expressed as $u=y / \Gamma$, with $y$ being the spatial coordinate perpendicular to the cement surface. An extended version of the above equations can be found elsewhere [8].

\section{Results and discussion}

Figure 1 displays the fit of the experimental curves for the rate of $\mathrm{C}-\mathrm{S}-\mathrm{H}$ precipitation, by means of the kinetic model outlined in the previous section. The model is capable of fitting the XRD data with excellent accuracy.
The curves displayed in figure 1 show a delay in the beginning of the acceleration stage, proportionally to the amount of PCE dosed, as well established in the literature.

The output from the model suggests a substantial switch from heterogeneous nucleation, to homogeneous nucleation in the presence of PCE. Specifically, the number of nuclei formed by heterogeneous nucleation per unit surface of substrate, as predicted by the model, decreases from $4.55 \times 10^{-11} \mathrm{~m}^{-2}$ to less than $3.00 \times 10^{-10} \mathrm{~m}^{-}$ ${ }^{2}$, with a majority of nuclei forming by homogeneous nucleation, whereas the number of homogeneous nuclei is negligible in the absence of PCE. However, the total number of nuclei formed is nearly halved in the presence of PCE. Such a hindered nucleation rate, in combination with a predicted decrease of the mean growth rate of $30-40 \%\left(3.50 \times 10^{-11} \mathrm{~m} / \mathrm{s}\right.$ for the blank paste to a minimum of $2.10 \times 10^{-11} \mathrm{~m} / \mathrm{s}$ in the presence of PCE) explains the net decrease in the overall kinetics of $\mathrm{C}_{3} \mathrm{~S}$ hydration.
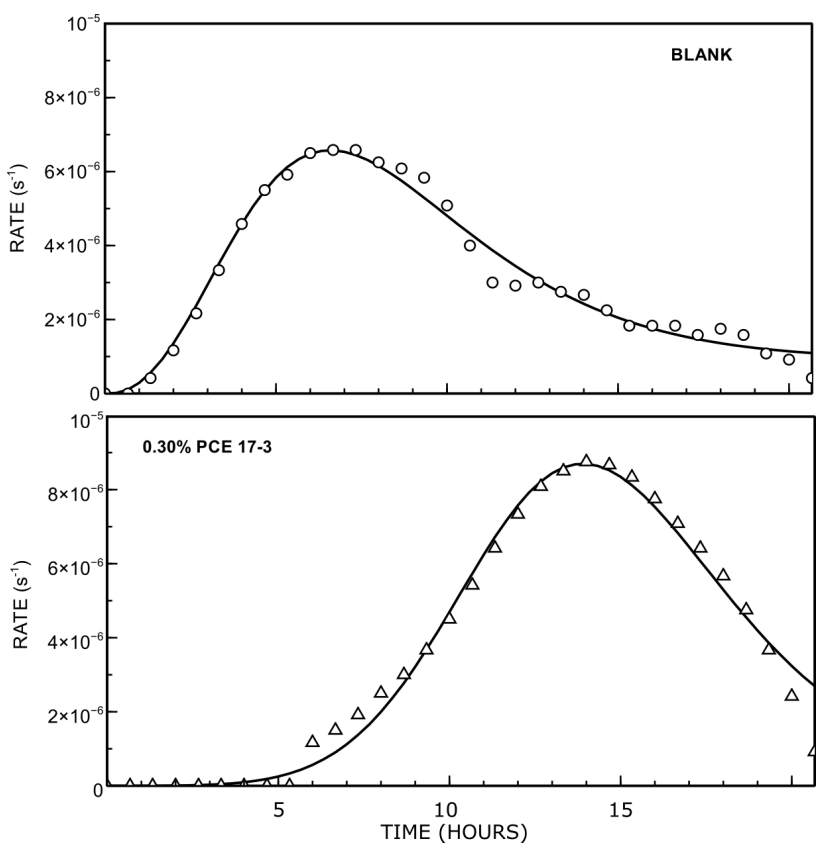

Fig. 1 Fitting of the XRD data (symbols, one in two data points are displayed with the aim of facilitating visualization) by means of the kinetic model (solid curve) for the $\mathrm{C}_{3} \mathrm{~S}$ paste in the absence (top) and in the presence (bottom) of PCE.

These results suggest that the nucleation and growth of $\mathrm{C}-\mathrm{S}-\mathrm{H}$ exerts a fundamental role in controlling the overall hydration kinetics and that PCE molecules induce a delay by acting on the modes and rates of C-S-H precipitation. The switch to homogeneous nucleation in the presence of PCE had been previously observed based on XRDtomography measurements [9].

Nonetheless, a better understanding of the action exerted by PCE molecules on hydration kinetics and microstructural development, will require a more refined understanding of the mechanisms of C-S-H precipitation, since classical models of heterogeneous/homogeneous nucleation and growth may be too idealized to provide an 
accurate picture. Some steps forward have been recently achieved by implementing more complex numerical models for the aggregation of C-S-H nano-particles [10]. A further advance will be obtained by implementing into such models alternative pathways to phase separation, which include the formation of pre-nucleation clusters [11]. Such models are especially relevant in the presence of organic molecules, such PCE [12].

\section{References}

1. F. Dalas, A. Nonat, S. Pourchet, M. Mosquet, D. Rinaldi, S. Sabio, Cem. Concr. Res. 67, 21-30 (2015)

2. G. Ferrari, L. Valentini, V. Russo, M.C. Dalconi, M. Favero, G. Artioli, Constr. Build. Mater.130, 83-91 (2017)

3. G. Xing, W. Wang, J. Xu, RSC Adv. 6,106921106927 (2016)

4. L. Valentini, M.C. Dalconi, M. Favero, G. Artioli, G. Ferrari, J. Am. Ceram. Soc. 98, 1259-1264 (2015)

5. L. Valentini, J. Appl. Crystallogr. 46, 1899-1902 (2013)

6. M. Avrami, J. Chem. Phys. 7, 1103-1112 (1939)

7. J.J. Thomas, J. Am. Ceram. Soc. 90, 3282-3288 (2007)

8. L. Valentini, M. Favero, M.C. Dalconi, V. Russo, G. Ferrari, G. Artioli, Cryst. Growth. Des. 16, 646-654 (2016)

9. G. Artioli, L. Valentini, M. Voltolini, M.C. Dalconi, G. Ferrari, V. Russo, Cryst. GrowthDes. 15, 20-23 (2015)

10. M. Delhorme, C. Labbez, M. Turesson, E. Lesiewska, C.E. Woodward, B. Jonsson, Langmuir32, 2058-2066 (2016)

11. T. Sowoidnich, T. Rachowski, C. Roessler, A. Voelkel, H.M. Ludwig, Cem. Concr. Res. 73, $42-50$ (2015)

12. C. Rodriguez-Navarro, L.G. Benning, Elements9, 203-209 (2013) 